\title{
SOME COMMUTATIVITY THEOREMS FOR ASSOCIATIVE RINGS WITH CONSTRAINTS INVOLVING A NIL SUBSET
}

\author{
MOHD. ASHRAF
}

\begin{abstract}
We first prove that a ring $R$ with unity 1 is commutalive if and only if for each $x$ in $R$ either $x$ is central or there exists a polynomial $f(t) \in Z[t]$ such that $x-x^{2} f(x) \in A$, where $A$ is a nil subset of $R$ (not necessarily a subring of $R$ ) and $R$ stisfies any one of the conditions $\left[x, x^{m} y-x^{p} y^{n} x^{q}\right]=0$ and $\left[x, y x^{m}-x^{p} y^{n} x^{q}\right]=0$ for all $x, y$ in $R$, where $m \geq 0, n>1, p \geq 0, q \geq 0$ are integers depending on pair of elements $x, y$. Further the same result has been extended for one sided s-unital rings. Finally a related result for a nil commutative subset $A$ is also obtained.
\end{abstract}

\section{Introduction.}

Let $A$ be a non-empty subset (not necessarily a subring) of an associative ring $R$ ( $R$ may be without unity 1 ); let $N, Z$ denote the set of nilpotent elements of $R$, the center of $R$ respectively. A ring $R$ is called right (resp. left) s-unital if $x \in x R$ (resp. $x \in R x$ ) for all $x$ in $R$. $R$ is called $s$-unital if $x \in x R \cap R x$ for all $x$ in $R$. $R$ is called normal if every idempotent of $R$ is central. The symbol $[x, y]$ stands for the commutator $x y-y x$, for any pair of elements of $R$. As usual $Z[t]$ is the totality of polynomials in $t$ with coefficients in $Z$, the ring of integers. We consider the following conditions:

(I-A) For each $x$ in $R$ there exists a polynomial $f(t) \in Z[t]$ such that $x-x^{2} f(x) \in A$.

(II-A) For each $x$ in $R$ either $x$ is central or there exists a polynomial $f(t) \in Z[t]$ such that $x-x^{2} f(x) \in A$.

(III-A) For each $x \in R$ and $a \in A,[[a, x], x]=0$.

(IV) For each $x, y$ in $R$ there exist integers $m=m(x, y) \geq 0, n=n(x, y)>1$. $p(x, y) \geq 0, q=q(x, y) \geq 0$ such that $\left[x, x^{m} y-x^{p} y^{n} x^{q}\right]=0$.

(V) For each $x, y$ in $R$ there exist integers $m=m(x, y) \geq 0, n=n(x, y)>1, p=$ $p(x, y) \geq 0, q=q(x, y) \geq 0$ such that $\left[x, y x^{m}-x^{p} y^{n} x^{q}\right]=0$.

A classicial theorem of Herstein [8] establishes commutativity of all rings satisfying (I-Z). Many authors have studied the commutativity of rings satisfying the condition (I-A), but always under some restrictions on $A$ (for a complete reference see [5]). Various

Received February 22, 1991.

AMS Subject classification (1980): 16A70. 
special cases of (IV) and (V) are shown to imply commutativity of rings (cf. [2], [3]) for example if the integral indices in the underlying conditions are 'global'. The objective of the present paper is to investigate the commutativity of $R$, when the integral indices are 'local' i.e. they are depending on pair of elements $x, y$ for their values. We establish commutativity of $R$, when (II-A) holds for a nil subset $A$ together with either of the conditions (IV) and (V). Moreover, the commutativity of ring $R$ satisfying (II-A) for a commutative nil subset $A$ has been studied. The results obtained here, improve the main theorems of the author et. al. [2] and [3].

\section{Property (II-A) For Noncommutative $A$}

Theorem 1. Let $R$ be a ring with unity 1 . The following statements are equivalent:

(i) $R$ is commulative.

(ii) $R$ satisfies either of the conditions $(I V) \&(V)$ and there exists a nil subset $A$ of $R$ for which $R$ satisfies (II-A).

For easy reference, we state the following well-known lemma.

Lemma 1 [1.0]. Let $R$ be a ring with unity 1 and $f: R \rightarrow R$ be a function such that $f(x)=f(x+1)$ holds for all $x$ in $R$. If for any $x$ in $R$, there exists a positive integer h such that $x^{h} f(x)=0$, then necessarily $f(x)=0$.

Proof of Theorem 1. Obviously (i) $\Longrightarrow$ (ii). Next, to show that (ii) $\Rightarrow$ (i) suppose that $a \in N$ and $x$ be an arbitrary element of $R$. If $R$ satisfies (IV), then there exist integers $m_{1} \geq 0, n_{1}>1, p_{1} \geq 0, q_{1} \geq 0$ depending on the pair of elements $x$ and $a$ such that $x^{m_{1}}[x, a]=x^{p_{2}}\left[x, a^{n_{1}}\right] x^{q_{1}}$. Again if we choose $m_{2} \geq 0, n_{2}>1, p_{2} \geq 0, q_{2} \geq 0$ depending on the pair of elements $x$ and $a^{n_{1}}$, then $x^{m_{2}}\left[x, a^{n_{1}}\right]=x^{p_{2}}\left[x,\left(a^{n_{1}}\right)^{n_{2}}\right] x^{q_{2}}$. Thus for any positive integer $k$ we have integers $m_{1}, m_{2}, \ldots, m_{k} \geq 0, n_{1}, n_{2}, \ldots, n_{k}>$ $1, p_{1}, p_{2}, \ldots, p_{k} \geq 0$ and $q_{1}, q_{2}, \ldots, q_{k} \geq 0$ such that

$$
\begin{aligned}
x^{m_{1}+m_{2}+\ldots+m_{k}}[x, a] & =x^{m_{2}+\ldots+m_{k}} x^{p_{1}}\left[x, a^{n_{1}}\right] x^{q_{2}} \\
& =x^{m_{3}+\ldots+m_{k}} x^{p_{1}+p_{2}}\left[x, a^{n_{2} n_{2}}\right] x^{q_{1}+q_{2}} \\
& =---\cdots \\
& =---\cdots \\
& =x^{p_{1}+p_{2}+\ldots+p_{k}}\left[x, a^{n_{1} n_{2} \ldots n_{k}}\right] x^{q_{1}+\ldots+q_{k}} .
\end{aligned}
$$

Hence $x^{m_{1}+m_{2}+\ldots+m_{k}}[x, a]=0$ for sufficiently large $k$ and $a \in Z$, by Lemma 1 . Thus $N \subseteq Z$ and in view of (II-A) $R$ satisfies (I-Z). IIence $R$ is commutative by IIerstein's theorem [8].

Again if $R$ satisfies (V), then by using the same arguments as above we get the required result.

Remarks 1 . The following example suggests that it is essential to retain any one of the conditions (IV) and (V) together with (II-A) in the hypotheses of above theorem in order to get the commutativity of $R$. 
Example 1. Let $R=\left\{a I+B / B=\left(\begin{array}{lll}0 & b & c \\ 0 & 0 & d \\ 0 & 0 & 0\end{array}\right), I=\left(\begin{array}{lll}1 & 0 & 0 \\ 0 & 1 & 0 \\ 0 & 0 & 1\end{array}\right), a, b, c, d \in\right.$ $G F(2)\}$. Obviously $N=\{B\}$ and $R$ satisfies neither (IV) nor (V). If we assume that $A=N$, then for any $x$ in $R, x-x^{2} f(x) \in A$. However, $R$ is not commutative.

2. The justification for unity 1 in the hypotheses of our theorem may be given by the following example.

Example 2. Let $R=\left\{\left(\begin{array}{lll}0 & a & b \\ 0 & 0 & c \\ 0 & 0 & 0\end{array}\right) / a, b, c \in G F(2)\right\}$. It can be easily seen that $R$ is a nilpotent ring of index 3. Thus $R=N$ and $R$ satisfies both the conditions (IV) $\&$ (V). Next, if we assume that $A=N$ then $R$ also satisfies condition (II-A). However, $R$ is not commutative.

3. Although, the above example strengthens the existence of unity 1 in the hypotheses of our theorem, nevertheless, the same result may be extended in a more general setting.

Theorem 2. Let $R$ be a left (resp. right) s-unital ring satisfying (IV) (resp. V). Suppose, further that there exists a subset $A$ of $N$ for which $R$ satisfies (II-A). Then $R$ is commutative (and conversely).

The following lemma is essentially proved in [13].

Lemma 2. Let $R$ be a right (resp. left) s-unital ring. If for each pair of elements $x, y$ of $R$ there exist a positive integer $k=k(x, y)$ and an element $e^{\prime}=e^{\prime}(x, y)$ of $R$ such that $e^{\prime} x^{k}=x^{k}$ and $e^{\prime} y^{k}=y^{k}$ (resp. $x^{k} e^{\prime}=x^{k}$ and $y^{k} e^{\prime}=y^{k}$ ), then $R$ is s-unital.

Proof of Theorem 2. Since $R$ is left (resp. right) $s$-untial then for any $x, y$ in $R$, we can find an element $e$ of $R$ such that $e x=x$ and $e y=y$ (resp. $x e=x$ and $y e=y$ ). Thus there exist integers $m=m(x, e) \geq 0, n=n(x, e)>1, p=p(x, e) \geq 0$ and $q=q(x, e) \geq 0$ such that

$$
\begin{aligned}
x^{m+1} e & =\left[x, x^{m} e-x^{p} e^{n} x^{q}\right]+x^{m+1}=x^{m+1} \\
\text { (resp. } \quad e x^{m+1} & =\left[x, e x^{m}-x^{p} e^{n} x^{q}\right]+e x^{m+1}=x^{m+1} \text { ). }
\end{aligned}
$$

Similarly, $y^{m^{\prime}+1} e=y^{m^{\prime}+1}$ (resp. $e y^{m^{\prime}+1}=y^{m^{\prime}+1}$ ). IIence $x^{m+m^{\prime}+1} e=x^{m+m^{\prime}+1}$, $y^{m+m^{\prime}+1} e=y^{m+m^{\prime}+1}$ (resp. $\left.e x^{m+m^{\prime}+1}=x^{m+m^{\prime}+1}, e y^{m+m^{\prime}+1}=y^{m+m^{\prime}+1}\right)$ and in view of Lemma $2, R$ is $s$-unital. Thus by [9, Proposition 1], we may assume that $R$ has unity 1 and hence $R$ is commutative by Theorem 1 .

Remark 4. The following example shows that there are noncommutative left (resp. right) s-unital rings satisfying (V) (resp. (IV)).

Example 3. Let

$$
R_{1}=\left\{\left(\begin{array}{ll}
0 & 0 \\
0 & 0
\end{array}\right),\left(\begin{array}{ll}
1 & 0 \\
1 & 0
\end{array}\right),\left(\begin{array}{ll}
0 & 1 \\
0 & 1
\end{array}\right),\left(\begin{array}{ll}
1 & 1 \\
1 & 1
\end{array}\right)\right\}
$$


and

$$
R_{2}=\left\{\left(\begin{array}{ll}
0 & 0 \\
0 & 0
\end{array}\right),\left(\begin{array}{ll}
1 & 1 \\
0 & 0
\end{array}\right),\left(\begin{array}{ll}
0 & 0 \\
1 & 1
\end{array}\right),\left(\begin{array}{ll}
1 & 1 \\
1 & 1
\end{array}\right)\right\}
$$

be two subrings of $2 \times 2$ matrices over $G F(2)$. Obviously in both the cases $N$ is the set consisting of the matrices $\left(\begin{array}{ll}0 & 0 \\ 0 & 0\end{array}\right),\left(\begin{array}{ll}1 & 1 \\ 1 & 1\end{array}\right)$. Further if $A=N$, then for any $x$ in $R$ we can find a polynomial $f(t)$, for example $f(t)=t$, such that $x-x^{2} f(x) \in A$. Also $R_{1}$ (resp. $R_{2}$ ) is a left (resp. right) $s$-unital ring and for any fixed integers $m \geq 0, n>$ $1, p \geq 0, q \geq 0, R_{1}$ (resp. $R_{2}$ ) satisfies the condition (V) (resp. (IV)).

\section{Property (II-A) For Commutative $A$}

Motivated by Theorem 1 of Tominaga and Yaqub [12], we derive the following:

Theorem 3. Let $R$ be a normal ring, and let $A$ be a nil commutative subset of $R$ for which $R$ satisfies $(I I-A)$. Then $R$ is commutative.

In the proof we shall use the following lemma, the proof of which is contained in that of $[11]$.

Lemma 3(i). Let $\phi$ be a ring homomorphism of $R$ onto $R^{*}$. If $R$ satisfies $(I-A)$, $(I I-A)$ or $(I I I-A)$, then $R^{*}$ satisfies $(I-\phi(A)),(I I-\phi(A))$ or $(I I I-\phi(A))$ respectively.

(ii). If there exists a commutative subset $A$ of $N$ for which $R$ satisfies (II-A) and (III-A), then $R$ is commutative.

(iii). If $A$ is commutative and $R$ satisfies $(I I-A)$, then $N$ is a commutative nil ideal of $R$ containing a commutator ideal of $R$ and contained in a centralizer of $A$, in particular, $N^{2} \subseteq Z$.

Proof of Theorem 3. In view of Lemma 3(i), $R$ can be assume to be subdiractly irreducible. Let $x$ be an arbitrary element of $R \backslash Z$. By using hypotheses (II-A), we find that there exists $y \in\langle x\rangle$ and a positive integer $m$ such that $x^{m}=x^{m+1} y$. Obviously, $e=x^{m} y^{m}$ is an idempotent with $x^{m}=x^{m} e$. Since idempotents of $R$ are central, hence $e$ is either 0 or 1. But $R$ has no unity, hence $e=0$ and by Lemma 3(iii) $x$ is in the commutative ideal $N$ and so $[[a, x], x]=0$ for all $a \in A$. Hence $R$ is commutative by Lemma 3(ii).

Remarks 6. Example 3 also shows that the condition (II-A) alone does not imply commutativity of rings in the above theorem.

7. In retrospect, it is tempting to conjecture as follows:

Conjecture. Let $R$ be a ring satisfying any one of the conditions (IV) and (V). Further, if there exists a nil commutative subset $A$ for which $R$ satisfies (II-A), then $R$ is commutative. 
8. A careful observation of the proof of Theorem 3 shows that the above conjecture is true if $R$ is normal. However, Example 3, violates the above conjecture because the centrality of idempotents in $R_{1}$ (resp. $R_{2}$ ) are not implied by the condition (V) (resp. (IV)) together with (II-A).

\section{Acknowledgement}

I would like to express my indebtedness and gratitude to the refree for his helpful suggestions and valuable comments.

\section{References}

[1] Mohd. Ashraf and M. A. Quadri, "On commutativity of associative rings with constraints involving a subset", Rad. Mat. 5, 141-149, 1989.

[2] Mohd. Ashraf, M. A. Quadri and Asma Ali, "On a commutativity property for rings", J. Indian Math. Soc. 55, 1-4, 1990.

[3] - "On commutativity of one sided s-unital rings", Rad. Mat. 6, 111-117, 1990.

[4] H. E. Bell, "A commutativity study for periodic rings", Pacific J. Math. 70, 29-36, 1977.

[5] - - "On two commutativity properties for rings", Math. Japonica 26 No. 5, 523-528, 1981.

[6] - - "On two commutativity properties for rings-II", Math. Japonica 27 No. 3, 319-325, 1982.

[7] I. N. Herstein, "A generalization of a theorem of Jacobson", Amer. J. Math. 73, 756-762, 1951.

[8] - - "The structure of a certain class of rings", A mer. J. Math. 75, 864-871, 1953.

[9] Y. Hiramo, Y. Kobayashi and H. Tominaga, "Some polynomial identities and commutativity of s-unital rings", Math. J. Okayama Univ. 24, 7-13, 1982.

[10] W. K. Nicholson and A. Yaqub, "A commutativity theorem for rings and groups", Canad. Math. Bull. 22, 419-123, 1979.

[11] H. Tominaga and A. Yaqub, "Some commutativity properties for rings", Math. J. Okayama Univ. $25,81-86,1983$.

[12] - "Some commutativity properties for rings-II", Math. J. Okayama Univ. 25, 173-179, 1983.

[13] —- "A commutativity theorem for one sided s-unital rings", Math. J. Okaya me Univ. 26, 125-128, 1984 .

Department of Mathematics, Aligarh Muslim University, Aligarh-202002, India. 


\title{
ROTARU STARLIKE INTEGRAI OPERATORS
}

\author{
SUBHAS S. BHOOSNURMATH AND S. R. SWAMY
}

\begin{abstract}
A bstract. Let $S^{*}(a, b)$ denote the class of analytic functions $f$ in the unit disc $U$, with $f(0)=f^{\prime}(0)-1=0$, satisfying the condition $\left|\left(z f^{\prime}(z) / f(z)\right)-a\right|<b, z \in U$, where $a \in C,|a-1|<b \leq \operatorname{Re}(a)$. In this paper we obtain integral operators which map $S^{*}(a, b)$ into $S^{*}(a, b)$ and $S^{*}(\lambda) \times S^{*}(a, b)$ into $S^{*}(\lambda)$.
\end{abstract}

\section{Introduction.}

Let $V$ denote the class of functions $f$ analytic in the unit disc $U$, with $f(0)=$ $f^{\prime}(0)-1=0$. A function $f$ of $V$ is said to belong to $S^{*}(\lambda)$, the class of starlike functions of order $\lambda$, if $\operatorname{Re}\left(z f^{\prime}(z) / f(z)\right)>\lambda$, for $z \in U, 0 \leq \lambda<1$. The class $S^{*}$ of starlike functions is identified by $S^{*}(0) \equiv S^{*}$. In [2], Rotaru investigated properties of the class $S^{*}(a, b)$ of functions $f \in V$ satisfying $\left|\left(z f^{\prime}(z) / f(z)\right)-a\right|<b, z \in U$, where $a \in C$, $|a-1|<b \leq \operatorname{Re}(a)$. It is clear that $S^{*}(a, b) \subset S^{*}(\operatorname{Re}(a)-b) \subset S^{*}$.

Recently in [3], Vinod kumar and Shukla have studied the integral operators of the form

$$
I(f)=\left[\frac{\gamma+\beta}{z^{\gamma}} \int_{0}^{z} t^{\delta-1} f^{\alpha}(t) d t\right]^{1 / \beta}
$$

and

$$
I(f, g)=\left[\frac{\gamma+\beta+\sigma}{z^{\gamma+\sigma}} \int_{0}^{z} t^{\delta-1} f^{\alpha}(t) g^{\sigma}(t) d t\right]^{1 / \beta}
$$

where $\alpha, \beta, \gamma, \delta$ and $\sigma$ are real constants and $f$ and $g$ belong to some favoured classes of univalent functions. By imposing suitable restrictions on $\alpha, \beta, \gamma, \delta$ and $\sigma$ thay have shown that, for $a=\bar{a}, I(f)$ maps $S^{*}(a, b)$ into itself and, for $a=\bar{a}, I(f, g)$ maps $S^{*}(\lambda) \times S^{*}(a, b)$ into $S^{*}(\lambda)$.

In the present paper we prove that, for $a \in C, I(f)$ maps $S^{*}(a, b)$ into itself and also, for $a \in C, I(f, g)$ maps $S^{*}(\lambda) \times S^{*}(a, b)$ into $S^{*}(\lambda)$.

Received February 22, 1991.

AMS (MOS) subject classification (1980); 30C45.

Keywords: Univalent, Starlike 


\section{Preliminary Lemmas}

The following lemma may be easily proved by using Schwarz's lemma as in Rotaru [2].

Lemma 2.1. The function $f$ belongs to $S^{*}(a, b)$ if and only if there exists a function $w$ analytic in $U$ satisfying $w(0)=0,|w(z)|<1$ for $z \in U$ such that

$$
\frac{z f^{\prime}(z)}{f(z)}=\frac{1+A w(z)}{1+\bar{B} w(z)}, \quad z \in U
$$

where $A=\left(b^{2}-|a|^{2}+a\right) / b$ and $B=(1-a) / b$.

Next we have the well known Jack's lemma [1].

Lemma 2.2. Let $w$ be analytic in $U$ with $w(0)=0$. If there exists a $z_{0} \in U$ such that $\max _{|z| \leq\left|z_{0}\right|}|w(z)|=\left|w\left(z_{0}\right)\right|$, then $z_{0} w^{\prime}\left(z_{0}\right)=k w\left(z_{0}\right)$ for some $k \geq 1$.

Lastly we prove a lemma which plays an important role in establishing our main result.

Lemma 2.3. Let $\alpha, \beta, b$ be real numbers and ' $a$ ' be a complex number sucl that $0<\alpha \leq \beta,|a-1|<b \leq \operatorname{Re}(a)$. If $d=(a \alpha+\beta-\alpha) / \beta$ and $e=b \alpha / \beta$, then $S^{*}(d, e) \subset$ $S^{*}(a, b)$.

Proof. We need only to consider the case $\alpha<\beta$. In order to establish the lemma it suffices to show that

$$
\operatorname{Re}(a)-b<\operatorname{Re}(d)-e \quad \text { and } \operatorname{Re}(d)+e<\operatorname{Re}(a)+b
$$

Let $\operatorname{Re}(a)-b \geq \operatorname{Re}(d)-e$. Then $\operatorname{Re}(a)-b \geq 1$, which is contrary to $|a-1|<b$. Next, suppose that $\operatorname{Re}(d)+e \geq \operatorname{Re}(a)+b$. Then $1 \geq \operatorname{Re}(a)+b$, which is also contrary to $|a-1|<b$. Therefore inequalities in (2.1) hold and hence the required result follows.

From now on $d$ and $e$ will be as in lemma 2.3 .

\section{Integral Operators That Map $S^{*}(a, b)$ into $S^{*}(a, b)$}

An integral operator which is defined on $S^{*}(a, b)$ and maps $S^{*}(a, b)$ into (onto) itself is called Rotaru starlike integral operator.

We now prove the following:

Theorem 3.1. Let $\alpha, \beta, \gamma$ and $\delta$ be real constants such that $0<\alpha \leq \beta$ and $\gamma+\beta=$ $\delta+\alpha$. If $f(z) \in S^{*}(a, b)$ then the function $F(z)$ defined by

$$
F(z)=\left[\frac{\gamma+\beta}{z^{\gamma}} \int_{0}^{z} t^{\delta-1} f^{\alpha}(t) d t\right]^{1 / \beta}
$$


also belongs to $S^{*}(a, b)$, provided $\frac{c}{4|\operatorname{Im}(a)|}>\gamma+\beta \geq \alpha c \frac{\operatorname{Re}(1-B)}{|1-B|^{2}}$, where $c=\left(b^{2}-|a-1|^{2}\right) / b$ and $B=(1-a) / b$.

Powers in (3.1) are meant as principal values.

Proof. First we prove that $F(z) \in S^{*}(d, e)$. Let $w(z)$ be a regular function defined in $U$ by

$$
\frac{z F^{\prime}(z)}{F(z)}=\frac{1+D w(z)}{1+\bar{E} w(z)}
$$

where $D=\left(e^{2}-|d|^{2}+d\right) / e, E=(1-d) / e$. Clearly $w(0)=0$ and $w(z) \neq-1 / \bar{E}$. From (3.1) and (3.2) we obtain

$$
N z^{\delta-\gamma} \frac{f^{\alpha}(z)}{F^{\beta}(z)}=\frac{N+M w(z)}{1+\bar{E} w(z)},
$$

where $N=\gamma+\beta, M=D \beta+\bar{E}_{\gamma}$. Logarithmic differentiation of (3.3) yields

$$
\frac{\alpha}{\beta}\left[\frac{z f^{\prime}(z)}{f(z)}-a\right]=\frac{E e+e w(z)}{1+\bar{E} w(z)}+\frac{m z w^{\prime}(z)}{(1+\bar{E} w(z))(N+M w(z))}
$$

where $m=\left(e^{2}-|d-1|^{2}\right) / e=\frac{\alpha}{\beta}\left(b^{2}-|a-1|^{2}\right) / b=\frac{\alpha}{\beta} c>0$.

Now we claim that $|w(z)|<1$, for otherwise by lemma 2.2 there exists a $z_{0} \in U$ such that $\left|w\left(z_{0}\right)\right|=1$ and

$$
z_{0} w^{\prime}\left(z_{0}\right)=k w\left(z_{0}\right), \quad k \geq 1
$$

From (3.4) and (3.5) we have

$$
\frac{\alpha}{\beta}\left[\frac{z_{0} f^{\prime}\left(z_{0}\right)}{f\left(z_{0}\right)}-a\right]=\frac{P\left(z_{0}\right)}{Q\left(z_{0}\right)}
$$

where

$$
P\left(z_{0}\right)=E e N+(E e M+e N+k m) w\left(z_{0}\right)+e M w^{2}\left(z_{0}\right)
$$

and

$$
Q\left(z_{0}\right)=N+(\bar{E} N+M) w\left(z_{0}\right)+\bar{E} M w^{2}\left(z_{0}\right)
$$

Clearly

$$
\begin{aligned}
& \left|P\left(z_{0}\right)\right|^{2}-e^{2}\left|Q\left(z_{0}\right)\right|^{2}>0 \quad \text { provided } \\
& \frac{k m}{2 e}+N+\operatorname{Re}(E M)+\operatorname{Re}\left((\bar{E} N+M) w\left(z_{0}\right)\right)>0
\end{aligned}
$$

or equivalently

$$
\frac{(k-1) m}{2 e}+A_{0}+B_{0} \operatorname{Re}\left(w\left(z_{0}\right)\right)-D_{0} \operatorname{Im}\left(w\left(z_{0}\right)\right)+\frac{m}{2 e}>0,
$$


where $A_{0}=\operatorname{Re}(N+E M), B_{0}=\operatorname{Re}(\bar{E} N+M)$ and $D_{0}=\operatorname{Im}(\bar{E} N+M)$. Since $k \geq 1$ and $m>0$, (3.8) holds if $A_{0} \pm B_{0} \geq 0$ and $\frac{m}{2 e} \pm D_{0}>0$.

Now

$$
\begin{aligned}
A_{0} \pm B_{0} & =\operatorname{Re}[\{(\gamma+\beta)+E(D \beta+\bar{E} \gamma)\} \pm\{\bar{E}(\gamma+\beta)+(D \beta+\bar{E} \gamma)\}] \\
& =\operatorname{Re}[(1 \pm \bar{E})(\gamma+\beta) \pm(1 \pm E)(D \beta+\bar{E} \gamma)] \\
& =\operatorname{Re}\left[|1 \pm E|^{2} \gamma+\beta(1 \pm E)(1 \pm D)\right] \\
& \geq 0, \text { provided } \gamma \geq-\frac{\beta \operatorname{Re}(1 \pm E)(1 \pm D)}{|1 \pm E|^{2}} \\
& =-\left(\beta \pm \alpha c \frac{\operatorname{Re}(1 \pm B)}{|1 \pm B|^{2}}\right), \text { where } B=(1-a) / b .
\end{aligned}
$$

and

$$
\frac{m}{2 e} \pm D_{0}=\frac{m}{2 e} \pm 2 \frac{(\gamma+\beta)}{e} \operatorname{Im}(d)>0 \text { provided } \gamma+\beta<\frac{m}{4|\operatorname{Im}(d)|}=\frac{c}{4|\operatorname{Im}(a)|}
$$
provided

Thus from (3.6) and (3.7) it follows in view of $e=\alpha b / \beta$ that $\left|\left(z_{0} f^{\prime}\left(z_{0}\right) / f\left(z_{0}\right)\right)-a\right|>b$

$$
\frac{c}{4|\operatorname{Im}(a)|}>\gamma+\beta \geq \alpha c \frac{\operatorname{Re}(1-B)}{|1-B|^{2}} .
$$

But this is contrary to the fact that $f \in S^{*}(a, b)$. Therefore $|w(z)|<1$ for $z$ in $U$. Thus from (3.2) and lemma 2.1, $F \in S^{*}(d, e)$. Hence from lemma 2.3, $F \in S^{*}(a, b)$.

Corollany 3.1. If $\frac{c}{4|\operatorname{Im}(a)|}>1 \geq a c \frac{\operatorname{Re}(1-B)}{|1-B|^{2}}$, where $c=\left(b^{2}-|a-1|^{2}\right) / b, B=$ $(1-a) / b$ and if $f \in S^{*}(a, b)$, then the function $F$ defined by

$$
F(z)=\left[z^{\beta-1} \int_{0}^{z}\left(\frac{f(t)}{t}\right)^{\alpha} d t\right]^{1 / \beta}
$$

also belongs to $S^{*}(a, b)$.

The above corollary follows by taking $\gamma=1-\beta$ and $\delta=1-\alpha$ in theorem 3.1.

Corollary 3.2. Let $\alpha$ and $\eta$ be real constants such that $\alpha>0, \eta \geq 0$. If $f \in S^{*}(a, b)$, then the function $F$ defined by

$$
F(z)=\left[\frac{\gamma+\alpha+\eta}{z^{\gamma}} \int_{0}^{z} t^{\gamma+\eta-1} f^{\alpha}(t) d t\right]^{1 /(\alpha+\eta)}
$$

also belongs to $S^{*}(a, b)$, provided $\frac{c}{4|\operatorname{Im}(a)|}>\alpha+\eta+\gamma \geq a c \frac{\operatorname{Re}(1-B)}{|1-B|^{2}}$, where $c=\left(b^{2}-\mid a-\right.$ $\left.\left.1\right|^{2}\right) / b$ and $B=(1-a) / b$.

The above result is obtained by setting $\beta=\alpha+\eta$ and $\delta=\gamma+\eta$ in theorem 3.1 .

Remark. For $a=\bar{a}$, theorem 3.1, corollary3.1 and corollary 3.2 of Vinod kumar and Shukla [3] are obtained as particular cases from our results. 
We now consider the integral operator defined in (3.1) in a limiting case. When $\alpha=\beta$, the relation (3.1) can be written as

$$
f(z)=F(z)\left(\left[\gamma+\left(\beta z F^{\prime}(z)\right) / F(z)\right] /(\gamma+\beta)\right)^{1 / \beta} .
$$

when $\beta \rightarrow 0$, the above relation reduces to

$$
f(z)=F(z) \exp \left[\left\{\left(z F^{\prime}(z) / F(z)\right)-1\right\} / \gamma\right]
$$

where $\gamma>0$. It follows from (3.9) that

$$
F(z)=f(z) \exp \left[-z^{-\gamma} \int_{0}^{z} t^{\gamma-1}\left\{t \frac{f^{\prime}(t)}{f(t)}-1\right\} d t\right]
$$

We now take $\gamma$ in (3.10) a complex number with $\operatorname{Re}(\gamma)>0$ and prove the following.

Theolem 3.2. If $f(z) \in S^{*}(a, b)$ and $\gamma \in C$ such that $\operatorname{Re}(\gamma)>0$, then the function $F$ defined by (3.10) also belongs to $S^{*}(a, b)$.

Proof. Let $w(z)$ be a regular function defined in $U$ by

$$
\frac{z F^{\prime}(z)}{F(z)}=\frac{1+A w(z)}{1+\bar{B} w(z)}
$$

where $A=\left(b^{2}-|a|^{2}+a\right) / b$ and $B=(1-a) / b$. Evidently $w(0)=0$ and $w(z) \neq-1 / \bar{B}$ for $z$ in $U$. Differentiating (3.9) and using (3.11) we get

$$
\frac{z f^{\prime}(z)}{f(z)}-a=\frac{b B+b w(z)}{1+\bar{B} w(z)}+\frac{(c / \gamma) z w^{\prime}(z)}{(1+\bar{B} w(z))^{2}}
$$

where $c=\left(b^{2}-|a-1|^{2}\right) / b>0$. We shall prove that $|w(z)|<1, z \in U$. For, if not, there exists a. $z_{0} \in U$, by lemma 2.2 , such that $\left|w\left(z_{0}\right)\right|=1$ and

$$
z_{0} w^{\prime}\left(z_{0}\right)=k w\left(z_{0}\right), \quad k \geq 1
$$

From (3.12) and (3.13) we obtain

$$
\frac{z_{0} f^{\prime}\left(z_{0}\right)}{f\left(z_{0}\right)}-a=b\left[\frac{B+w\left(z_{0}\right)+\Phi\left(z_{0}\right) w\left(z_{0}\right)}{1+\bar{B} w\left(z_{0}\right)}\right]
$$

where $\Phi\left(z_{0}\right)=\frac{k c}{b \gamma\left(1+\bar{B} w\left(z_{0}\right)\right.}$

Now $\left|\frac{z_{0} f^{\prime}\left(z_{0}\right)}{f\left(z_{0}\right)}-a\right|>b$ provided $\left|B+w\left(z_{0}\right)+\Phi\left(z_{0}\right) w\left(z_{0}\right)\right|^{2}>\left|1+\bar{B} w\left(z_{0}\right)\right|^{2}$. This condition reduces to the following:

$$
\left|\Phi\left(z_{0}\right)\right|^{2}+2 \operatorname{Re}\left[\left(1+\bar{B} w\left(z_{0}\right) \Phi\left(z_{0}\right)\right]>0,\right.
$$


which is true since $\operatorname{Re}\left[1+\bar{B} w\left(z_{0}\right) \Phi\left(z_{0}\right)\right]=\operatorname{Re}\left(\frac{k c}{b \gamma}\right)>0$. But this is a contradiction to the hypothesis that $f \in S^{*}(a, b)$. Hence $|w(z)|<1$ for $z \in U$ and from (3.11) we conclude that $F \in S^{*}(a, b)$.

Remark. For $a=\bar{a}$, the above theorem improves a recent result of Vinodkumar and Shukla [3] who proved it when $\gamma$ is a real number. Here it is worth noting that the technique used by them with the help of Jack's lemma, fails when $\gamma$ is a complex number.

\section{Integral Operators that Map $S^{*}(\lambda) \times S^{*}(a, b)$ into $S^{*}(\lambda)$}

Theorem 4.1. Let $\alpha, \beta, \gamma, \delta$ and $\sigma$ be real constants such that $\alpha>0, \beta \geq \alpha, \sigma \geq 0$, $\alpha+\delta=\beta+\gamma$ and $\gamma+\sigma+\lambda \beta>0$. If $f \in S^{*}(\lambda)$ and $g \in S^{*}(a, b),(a, b) \in R=\{(a, b)$ : $\left.|a-1|<b \leq a^{*}\right\}$, where $a^{*}=\min \left\{\operatorname{Re}(a),(\operatorname{Re}(a)-1)+\frac{\beta(1-\lambda)}{2 \sigma(\gamma+\sigma+\lambda \beta)}\right\}$, then the function $F$ defined by

$$
F(z)=\left[\frac{\gamma+\beta+\sigma}{z^{\gamma+\sigma}} \int_{0}^{z} t^{\delta-1} f^{\alpha}(t) g^{\sigma}(t) d t\right]^{1 / \beta}
$$

also belongs to $S^{*}(\lambda)$.

Powers in (4.1) are meant as principal values.

Proof. Define a regular function $w(z)$ in $U$ by

$$
\frac{z F^{\prime}(z)}{F(z)}=\frac{1+(2 \lambda-1) w(z)}{1+w(z)} \text {. }
$$

Clearly $w(0)=0$ and $w(z) \neq-1$ in $U$. From (4.1) and (4.2) we have

$$
N_{1} z^{\delta-\gamma-\sigma}\left\{\frac{f^{\alpha}(z) g^{\sigma}(z)}{F^{\beta}(z)}\right\}=\frac{N_{1}+M_{1} w(z)}{1+w(z)},
$$

where $N_{1}=\gamma+\beta+\sigma$ and $M_{1}=\gamma+\sigma-\beta+2 \lambda \beta$.

Logarithmic differentiation of (4.3) yiclds

$$
\begin{aligned}
\frac{z f^{\prime}(z)}{f(z)}= & \frac{\sigma}{\alpha}(1-a)-\frac{\sigma}{\alpha}\left\{\frac{z g^{\prime}(z)}{g(z)}-a\right\}+\frac{\beta}{\alpha}\left\{\frac{1+(2 \dot{\lambda}-1) w(z)}{1+w(z)}\right\} \\
& -\left\{\frac{\beta-\alpha}{\alpha}\right\}-\frac{2 \beta(1-\lambda) z w^{\prime}(z)}{\alpha(1+w(z))\left(N_{1}+M_{1} w(z)\right)} .
\end{aligned}
$$

Now we claim that $|w(z)|<1$, for otherwise by lcmma 2.2 there exists a $z_{0} \in U$ such that $\left|w\left(z_{0}\right)\right|=1$ and

$$
z_{0} w^{\prime}\left(z_{0}\right)=k w\left(z_{0}\right), \quad k \geq 1 .
$$

Using the technique similar to the one employed in the proof of theorem 4.1 of Vinod kumar and Shukla [3], we obtain from (4.4) and (4.5)

$$
\begin{aligned}
\operatorname{Re}\left\{z_{0} \frac{f^{\prime}\left(z_{0}\right)}{f\left(z_{0}\right)}\right\} & \leq \lambda+\frac{2(\gamma+\sigma+\lambda \beta)\{2 \sigma(\gamma+\sigma+\lambda \beta)[b-(\operatorname{Re}(a)-1)]-\beta(1-\lambda)\}}{\alpha\left[N_{1}^{2}+2 N_{1} M_{1} \operatorname{Re}\left(w\left(z_{0}\right)\right)+M_{1}^{2}\right]} \\
& \leq \lambda, \text { provided } b \leq(\operatorname{Re}(a)-1)+\frac{\beta(1-\lambda)}{2 \sigma(\gamma+\sigma+\lambda \beta)} .
\end{aligned}
$$


But this is contrary to the fact that $f \in S^{*}(\lambda)$. Therefore $|w(z)|<1$ and hence, from (4.2), $F \in S^{*}(\lambda)$.

\section{References}

[1] I. S. Jack, "Functions starlike and convex of order $\alpha$ ", J. London Math. Soc., 2 (3), 469-479, 1971.

[2] P. Rotaru, "Subclasses of starlike functions", Mathematica, 29 (52), 2, 183-191, 1987.

[3] Vinod kumar and S. L. Shukla, "Jakubowski starlike integral operators", J. Austral Math. Soc. (Series A), 37, 117-127, 1984 .

Department of Mathematics, Karnatak University, Dharwad-580 003, India.

Department of Mathematics, J. M. Institute of Technology, Chitradurga-577 502, India. 